\title{
Nonarteritic anterior ischemic optic neuropathy associated with chronic anemia: a case series of myelodysplastic syndrome patients
}

\author{
Dimitrios Brouzas \\ Antonios Charakidas \\ loannis Ladas \\ Michael Apostolopoulos \\ Department of Ophthalmology, \\ University of Athens, Athens, Greece
}

\begin{abstract}
Introduction: We report on three cases of visual loss due to nonarteritic anterior ischemic optic neuropathy that developed during the course of refractory anemia, a subtype of myelodysplastic syndrome.

Patients and methods: Patients underwent fundus, visual field examination, and fluorescein angiography. A thrombophilic tendency investigation including prothrombin time, partial thromboplastin time, protein $\mathrm{C}$, free protein $\mathrm{S}$, and antithrombin III, polymerase chain reaction and hybridisation to allele-specific oligonucleotide probes and a bone marrow biopsy were also performed.

Results: Relative recovery of visual function was noted in two patients, a 58-year-old man and a 67-year-old woman, whereas the vision of the third patient, a 62-year-old man, showed only marginal improvement during the follow-up period. Two patients received vigorous blood transfusion during hospitalization, while dosage adjustment of the erythropoietin infusion was decided for the third one. Thrombophilic tendency was not identified in any patient.

Discussion: Chronic anemia, as presented in myelodysplastic syndrome's refractory anemia subtype, probably in the presence of additional factors, such as hypotension, is likely to be complicated by optic neuropathy, possibly through a mechanism of anemic hypoxia and/or microvascular insufficiency.
\end{abstract}

Keywords: myelodysplastic syndrome, anemia, optic neuropathy

\section{Introduction}

Nonarteritic ischemic optic neuropathy (NAION) typically occurs as a unilateral process in older patients who have vasculopathic risk factors, ${ }^{1}$ with rapid onset, stable course and generally poor recovery. The most commonly proposed pathogenic theory involves an insufficiency of the optic disc circulation, exacerbated by structural "crowding" of nerve fibers and supporting structures at the nerve head, resulting in inadequate oxygenation, ischemia and swelling of the disc. An altitudinal field defect, usually involving the inferior field, is most common, but generalized depression, broad arcuate scotomas, and cecocentral defects also are seen. ${ }^{2}$

The myelodysplastic syndromes (MDS) are a heterogeneous group of stem cell malignancies characterized by dysplastic and ineffective blood cell production and a variable risk of transformation to acute leukaemia. ${ }^{3}$ We report on three cases of nonarteritic anterior ischemic optic neuropathy (NAION), which developed during the course of refractory anemia, one of the morphological subtypes of MDS. ${ }^{4}$

\section{Case reports Case I}

A 58-year-old man presented with left-sided painless blurring of vision of one-week duration. His medical and ophthalmic history was unremarkable but he complained of 
moderate malaise and fatigue of recent onset. Best-corrected visual acuity was 20/50 OD and 20/150 OS. Mild nuclear cataract was noted bilaterally. A relative afferent pupillary defect was present in the left eye. Fundus examination revealed sectorial optic disc edema in the left eye, which corresponded to optic disc filling delay and late leakage on fluorescein angiography (Figure 1) as well as a related broad inferior-nasal arcuate scotoma. The erythrocyte sedimentation rate was $20 \mathrm{~mm}$ (1st hour) and C-reactive protein was within normal range. A full blood count showed profound anemia (hemoglobin $55 \mathrm{~g} / \mathrm{l})$, and moderate leucopenia $\left(4.9 \times 10^{9} / \mathrm{lt}\right)$ and thrombocytopenia $\left(114 \times 10^{9} / \mathrm{lt}\right)$. Blood pressure was 90/65 mmHg, pulse 105/min. A carotid Doppler study was normal. Further investigation in search of a thrombophilic tendency, including prothrombin time, partial thromboplastin time, protein $\mathrm{C}$, free protein $\mathrm{S}$, and antithrombin III yielded normal values. Polymerase chain reaction and hybridisation to allele-specific oligonucleotide probes (ViennaLab Labordiagnostika GmbH, Vienna, Austria) did not identify prothrombin, factor V or MTHFR thrombophilic mutations.
A diagnosis of anterior ischemic optic neuropathy was made and the patient was referred to the Internal Medicine Department for further evaluation. A bone marrow biopsy was suggestive of myelodysplasia (subtype refractory anemia). ${ }^{4}$ Transfusions of packed red cells, initiated two weeks following the approximate date of initiation of symptoms, raised hemoglobin to $98 \mathrm{~g} / \mathrm{l}$, and were followed by biweekly transfusions and initiation of erythropoietin in order to maintain hemoglobin over $100 \mathrm{~g} / \mathrm{l}$.

Within 10 days following admission, the visual acuity of the left eye had improved to 20/50 and remained stable for the rest of a six-month follow-up period, during which sectorial left optic disc pallor ensued.

\section{Case 2}

A 67-year-old woman presented with a five-day history of painless visual impairment in the left eye. Her medical history included chronic renal failure of unknown etiology controlled with hemodialysis, hypothyroidism and myelodysplastic syndrome (classified as refractory anemia) ${ }^{4}$ diagnosed
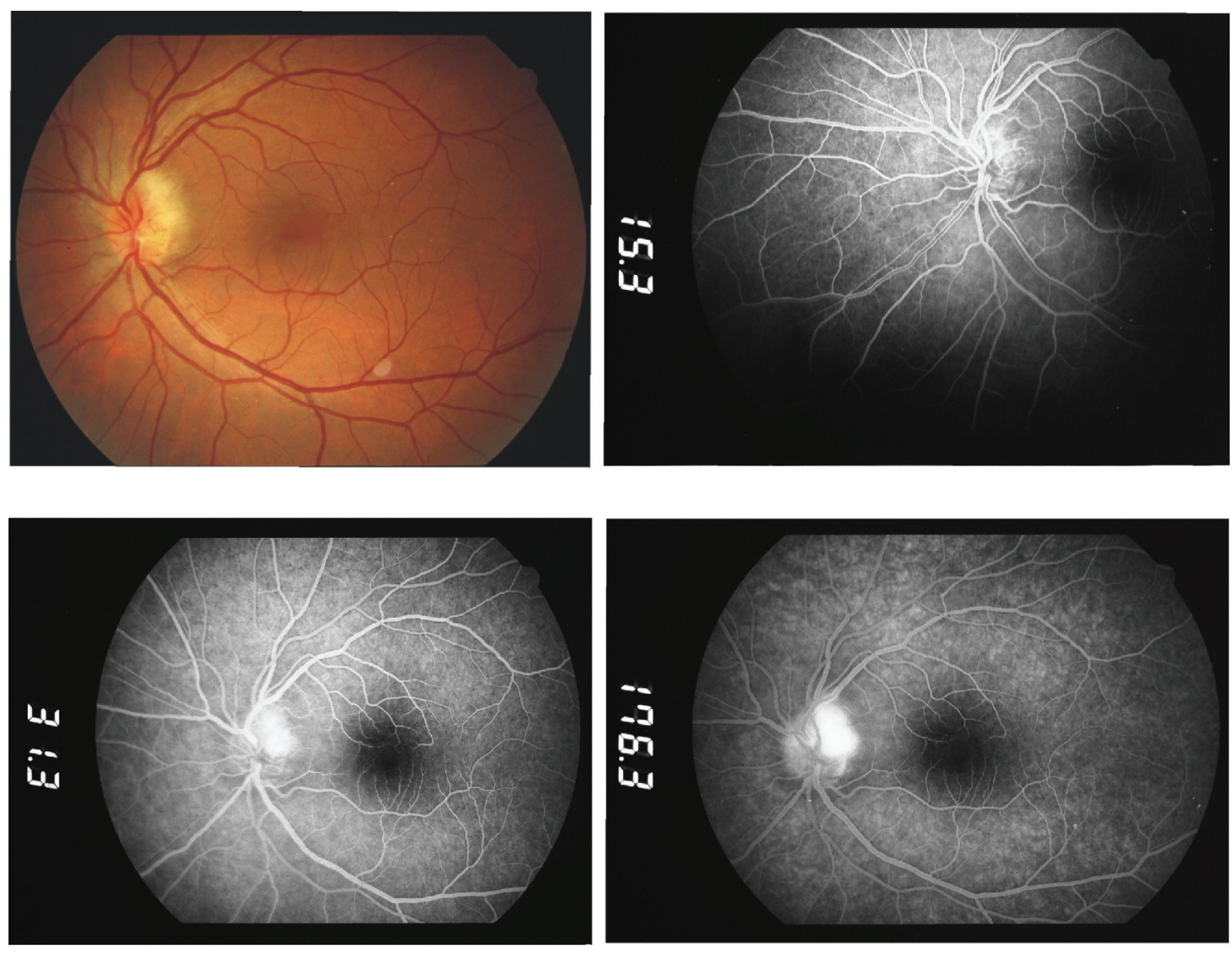

Figure I Case I on presentation. Color fundus photo and early, intermediate, and late fluorescein angiograms depicting optic disc edema and leakage at late stages. 
three months earlier. The patient was on thyroid hormone substitution therapy but erythropoietin was not prescribed, as an earlier attempt had resulted in the thrombotic occlusion of her arteriovenous fistula.

On admission, the best-corrected visual acuity was 20/50 OD and 20/800 OS. A left-sided relative afferent pupillary defect was present. Apart from bilateral mild senile cataract, anterior segment examination was unremarkable. Funduscopy and fluorescein angiography revealed an optic nerve head edema in the left eye, corresponding to visual field generalized depression.

Erythrocyte sedimentation rate was $17 \mathrm{~mm}$ (1st hour). C-reactive protein was found within normal range. On initial work-up, hemoglobin was $79 \mathrm{~g} / 1$, white blood cell count $11.3 \times 10^{9} /$ lt and platelets $307 \times 10^{9} /$ lt. Transfusion of packed red cells was initiated on the second day of admission in order to increase hemoglobin levels. Blood pressure was within normal range throughout hospitalization. Carotid bruits
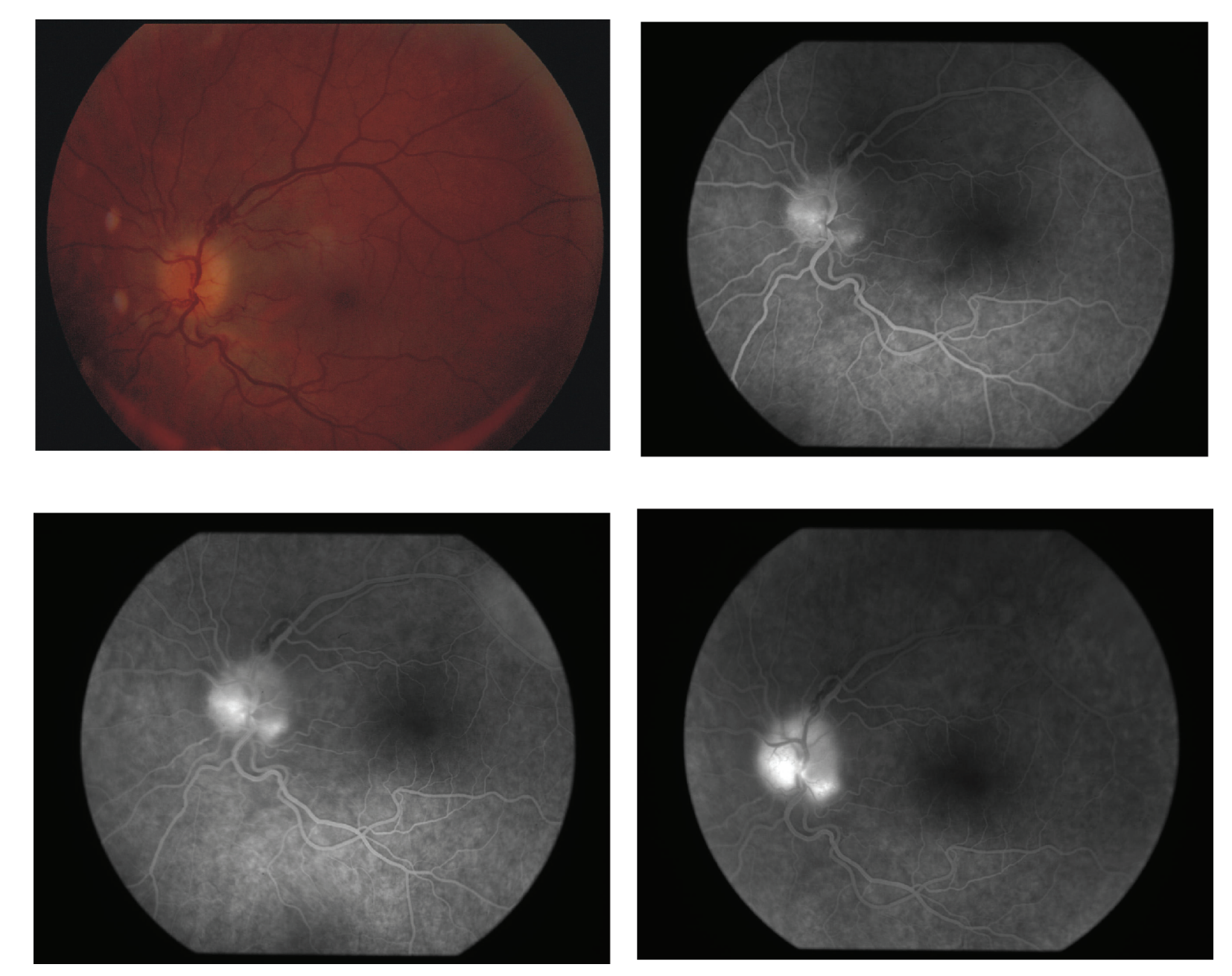

were not noted and a Doppler study showed normal carotid blood flow.

Within one week of hospitalization, visual acuity improved to 20/60 OS and remained stable for the rest of the follow-up period ( 6 months). During this period visual fields also improved and pallor of the left optic disc was noted.

\section{Case 3}

A 62-year-old man presented with painless reduction of visual acuity in the left eye that developed during the course of a lower respiratory tract infection a few days earlier. His medical history included myelodysplastic syndrome (classified as refractory anemia) ${ }^{4}$ diagnosed three years earlier, in the course of the laboratory investigation for chronic malaise and fatigue. The patient was put on an erythropoietin regimen that had increased his pre-treatment hematocrit from $27 \%$ to $34.5 \%$.

Figure 2 Case 3 on presentation. Color fundus photo and fluorecein angiograms.

Figure 2 Case 3 on presentation. Color fundus photo and fluorecein angiograms. 
On admission, the best-corrected visual acuity was 20/50 OD and 20/400 OS. A left-sided relative afferent pupillary defect was present and, apart from bilateral mild senile cataract, anterior segment examination was unremarkable. Funduscopy and fluorescein angiography revealed a left-sided optic nerve head edema (Figure 2), corresponding to superior altitudinal field defect and generalized depression.

Erythrocyte sedimentation rate was $17 \mathrm{~mm}$ (1st hour). C-reactive protein was found within normal range. On initial work-up, hematocrit was $30.7 \%$, white blood cell count $4.3 \times 10^{9} / 1 \mathrm{t}$ and platelets $307 \times 10^{9} / \mathrm{lt}$. Blood pressure was $130 / 70 \mathrm{mmHg}$ and remained within normal range throughout hospitalization. Dosage adjustment of his erythropoietin infusion rather than transfusion of packed red cells was preferred by the hematologist.

Within one week, visual acuity slightly improved to 20/200 OS and remained stable for the rest of the follow-up period ( 6 months). The visual fields remained severely constricted and atrophy of the left optic disc was noted.

\section{Discussion}

Hypertension, diabetes mellitus, and nocturnal hypotension have all been associated with the initiation of non-arteritic anterior ischemic optic neuropathy, ${ }^{1}$ with a congenitally anomalous optic disc with a small optic cup being an additional important risk factor. ${ }^{5}$ Acute anemia and hypotension are also known to be linked with shock-induced ischemic optic neuropathy in the course or immediately after massive gastrointenstinal hemorrhage and major surgical procedures. ${ }^{6,7}$

Although neuronal tissue generally meets its oxygen requirement even with profound anemia, there is evidence that the presence of an additional factor may induce neuronal tissue dysfunction. Chronic anemic patients with carotid stenosis, for example, have demonstrated transient neurological deficits during intermittent reductions of hemoglobin below a critical level. ${ }^{8}$ Similarly, children with generally well-tolerated iron deficiency anemia have presented with an ischemic stroke when a metabolic stress could not be met. ${ }^{9}$ Moreover chronic anemia due to repeated gastrointestinal bleeding has been reported to cause bilateral optic nerve infarction in the presence of acute hypotension. ${ }^{10}$

The refractory anemia subtype of MDS, is likely to be involved in the initiation of NAION through a mechanism of anemic hypoxia and/or microvascular insufficiency. Our first case, an otherwise healthy man, presented with hypotension (maximum blood pressure 90/65 $\mathrm{mmHg}$ ), whereas the second patient reported a history of hypothyroidism and chronic renal failure, which may induce episodes of hypotension, especially following hemodialysis sessions. The association of NAION with hypotension in the setting of renal failure and hemodialysis was first reported by Servilla and Groggel in $1986,{ }^{11}$ whereas Haider et al detected four cases of NAION after a 24-month follow-up of 60 patients under dialysis and implicated hypotension in one case, severe anemia in another one and generalized atherosclerosis in two patients. ${ }^{12}$ Although, theoretically, hypotension alone could trigger the manifestation of NAION in cases 1 and 2, our third patient - otherwise hematologically stable-developed optic neuropathy during a febrile respiratory infection that involved oxygen saturation changes.

The course of nonarteritic anterior ischemic optic neuropathy varies considerably. In most patients there is no further loss of vision although, in a small percentage, visual loss continues for several weeks. On the other hand, the Ischemic Optic Neuropathy Decompression Trial Research Group reports spontaneous improvement of visual acuity in $23.8 \%$ of patients and of visual fields in a further $24 \%$, comprising as many as $42.7 \%$ of NAION cases. ${ }^{13,14}$ In this context, the contribution of vigorous transfusion in the observed relative recovery of visual function of the two patients cannot be determined.

An alternative approach to the disease process would implicate a mechanism of vasculitis of the short posterior ciliary arteries. Small vessel vasculitis, of unclear pathogenesis so far, reportedly complicates approximately $10 \%$ of MDS and presents as cutaneous vasculitis and lupus-like syndromes. ${ }^{15,16}$ Our patients, however, did not report previous vasculitic manifestations and their erythrocyte sedimentation rate and C-reactive protein were normal.

This case series implies that the chronic anemia of myelodysplastic syndrome's refractory anemia subtype may be associated with the development of non-arteritic anterior ischemic optic neuropathy, presumably in the presence of an additional factor, such as hypotension, chronic renal failure, or metabolic disorder. Further research aiming to determining the odds ratio of developing NAION in association with refractory anemia MDS would place this link in perspective and justify the monitoring of these patients for a major opththalmic complication.

\section{Disclosure}

The authors report no conflicts of interest in this work.

\section{References}

1. Hayreh SS, Joos KM, Podhajsky PA, et al. Systemic diseases associated with nonarteritic anterior ischemic optic neuropathy. Am J Opththalmol. 1994;118:766-780.

2. Arnold AC. Ischemic optic neuropathy, diabetic papillopathy, and papillophlebitis. In: Myron Yannoff M, Duker JS, Editors. Ophthalmology. Philadelphia, PA, Mosby; 1999; pp. 11.7.1-11.7.6. 
3. List AF, Doll DC. The myelodysplastic syndromes In: Lee GR, Foerster J, Lukens J, et al. editors. Wintrobe's Clinical Hematology, 10th Edition. Philadelphia, PA: Lea and Febiger; 1998. pp. 2320-2341.

4. Bennet JM, Catovsky D, Daniel MT, et al. Proposals for classification of the MDS. Br J Haematol. 1982;51:189-199.

5. Beck RW, Savino PJ, Repka MX, et al. Optic disc structure in anterior ischemic optic neuropathy. Ophthalmology. 1984;91:1334-1337.

6. Jaben SL, Glaser JS, Daily M. Ischemic optic neuropathy following general surgical procedures. J Clin Neuroophthalmol. 1983;3:239-244.

7. Hollenhorst RW, Wagener HP. Loss of vision after distant hemorrhage. Am J Med Sci. 1950;219:209-244.

8. Shahar A, Sadeh M. Severe anaemia associated with transient neurological deficits. Stroke. 1991;22:1201-1202.

9. Hartfield DS, Lowry NJ, Keene DL, et al. Iron deficiency: A cause of stroke in infants and children. Pediatr Neurol. 1997;16:50-53.

10. Johnson MW, Kincaid MC, Trobe JD. Bilateral retrobulbar optic nerve infarctions after blood loss and hypotension. A clinicopathological case study. Ophthalmology. 1987;94:1577-1584.
11. Servilla K, Groggel G. Anterior ischemic optic neuropathy as a complication of hemodialysis. Am J Kidney Dis. 1986;8:61-63.

12. Haider S, Astbury N, Hamilton D. Optic neuropathy in uraemic patients on dialysis. Eye. 1993;7:148-151.

13. The Ischemic Optic Neuropathy Decompression Trial Research Group. Optic nerve decompression surgery for nonarteritic anterior ischemic optic neuropathy (NAION) is not effective and may be harmful. JAMA. 1995;274:612-632.

14. Arnold A, Hepler R. Natural history of nonarteritic anterior ischemic optic neuropathy. Neuroophthalmol. 1994;14:66-69.

15. Pirayesh A, Verbunt RJAM, Kluin PHM, Meinders AE, De Meijer PHEM. Myelodysplastic syndrome with vasculitic manifestations. J Intern Med. 1997;242:425-431.

16. Billstroem R, Johansson H, Johansson B, Mitelman F. Immune-mediated complications in patients with myelodysplastic syndromes - clinical and cytogenetic features. Eur J Haematol. 1995;55:42-48. 
